\title{
Efficacy of musculoskeletal injections given by primary care providers in the office
}

This article was published in the following Dove Press journal:

International Journal of General Medicine

13 September 2013

Number of times this article has been viewed

\section{Tasnim Eghbal Eftekhaari' Mirzaali Nazarnezhad' Iman Ghasemzadeh ${ }^{2}$ \\ 'Clinical Research Development Center, Hormozgan University of Medical Sciences Bandar Abbas, Iran; ${ }^{2}$ Infectious Disease Research Center Hormozgan University of Medical Sciences, Bandar Abbas, Iran}

Correspondence: Tasnim Eghbal Eftekhaari Clinical Research Development Center, Hormozgan University of Medical Sciences, Shahid Mohammadi Hospital,

Bandar Abbas, Iran

Zip Code 7919915519

Tel/Fax +98 76 I333 7/92

Email tasnim.eftekhaari@gmail.com

\section{Dear editor}

Patients commonly present to primary care physicians with musculoskeletal symptoms. Clinicians qualified in internal medicine must be knowledgeable about the diagnosis and management of musculoskeletal diseases, yet they often receive inadequate postgraduate training on this topic. The musculoskeletal problems most frequently encountered in our busy injection practice involve, in decreasing order, the knees, trochanteric bursae, and glenohumeral joints. ${ }^{1}$ However, patients usually present to a primary care physician and embark on treatment there, despite having the option of referral to a subspecialist fully trained in this procedure.

A retrospective cohort study conducted in patients with musculoskeletal pain treated with corticosteroid injections by primary care providers compared quality of life, procedural benefit, and patient satisfaction before and after musculoskeletal injections in the office setting. ${ }^{2}$ This paper indicated that musculoskeletal corticosteroid injections were performed by a range of providers, including orthopedic surgeons, rheumatologists, psychiatrists, podiatrists, and primary care physicians (both family and internal medicine). ${ }^{2}$ The study included patients older than 18 years with bursitis of the knee, shoulder, or trochanteric sites treated by primary care practitioners in the office setting. $^{2}$

Exclusion criteria were:

- palindromic rheumatoid arthritis, which may be confused with fibromyalgia, sudden onset of polyarthritis, or periarticular tissue, for which gold is the standard of care $^{3}$

- Patellofemoral pain syndrome, ie, peripatellar tendinitis or bursitis, plica syndromes, Sinding Larsen's disease, Osgood Schlatter's disease, neuromas, and other rarely occurring pathologies; it has been suggested that patients with a clinical presentation of anterior knee pain may have patellofemoral pain syndrome, and recent studies advocate a comprehensive treatment approach allowing for individualized treatment. ${ }^{4}$

Of note, there was no mention in this paper of shoulder pain treated with manual or manipulative therapy, which should have been a further exclusion criterion because, if misdiagnosed as adhesive capsulitis, manipulation may aggravate the condition, making interventional treatment necessary.

Another condition that should be mentioned is greater trochanteric pain syndrome, which is a regional syndrome characterized by pain and reproducible tenderness in 
the region of the greater trochanter, buttock, and/or lateral thigh, and may mimic the symptoms of lumbar nerve root compression. Despite these features being well described, the diagnosis of greater trochanteric pain syndrome is often missed, and documentation of its prevalence in specialist orthopedic practice is lacking. This condition, as reported by Tortolani et al, ${ }^{6}$ is often misdiagnosed by orthopedic surgeons, so it is unlikely that a primary care physician with limited training and no access to ultrasound facilities could diagnose the disease accurately and treat it appropriately. Treatment often requires a mixture of corticosteroid and analgesic injections and severe cases may require lumbar decompression. Mild to moderate cases often respond to injections if correctly diagnosed, but surgical intervention is usually required in the later stages. ${ }^{6}$

A study performed in University of Chicago reported that $67 \%$ of residents and faculty responded that articular pain should be treated by corticosteroid injection, but only $19 \%$ of them practiced this themselves. Eighty nine percent of primary care physicians referred the patients to sub-specialists mainly because of the pain associated with the procedure, and only $9.6 \%$ of these patients were referred to primary care physician. ${ }^{7}$

In a prospective study, 44 patients were randomly assigned to receive shoulder manipulation and arthroscopic arthrolysis $(n=23)$ or glenohumeral steroid injections $(n=21)$. Patients were followed up at 3, 6, and 12 weeks, then at 6 and 12 months using the Constant-Murley, American Shoulder and Elbow Surgeons, Shoulder Rating Scale of the University of California Los Angeles, and Simple Shoulder Test evaluation scales. Ranges of passive forward flexion, abduction, and internal and external rotation were also recorded. Both types of treatment were effective in improving the final range of motion; however, patients randomized to shoulder manipulation and arthroscopic arthrolysis accomplished their goal at 6 weeks whereas those randomized to glenohumeral steroid injections did not do so until 12 weeks, indicating that shoulder manipulation has a more rapid therapeutic effect than steroid injections. ${ }^{8}$

A further study was designed to evaluate whether a combination of shoulder exercises and ultrasound-guided injection into the bursa significantly improves the treatment outcome in adhesive bursitis. ${ }^{9}$ Seventy patients were allocated to ultrasound-guided injection $(n=35)$ or to ultrasound-guided injection and a home exercise program for 1 month $(n=35)$. Pain and shoulder function were assessed before and at 1 and 3 months after treatment. No differences were found on functional assessment. The combination of ultrasound-guided injection and shoulder exercises in the treatment of subacromial adhesive bursitis was effective in achieving complete pain relief in the medium term, indicating that ultrasound-guided injection is effective, but few primary care physicians use ultrasound in their routine practice.

Another issue is the common misconceptions about the causes of lateral hip pain and tenderness, which frequently lead to therapeutic strategies providing only temporary solutions rather than addressing the underlying pathology. Trochanteric bursitis is frequently implicated, but is seldom the primary cause of pain in chronic cases, so it is important to address hip rotator cuff tendinopathy and pelvic core instability. Treatment options include exercise, physical modalities, corticosteroid injections, extracorporeal shock wave therapy, and regenerative injection therapies, with surgery reserved for recalcitrant cases. By understanding the anatomy of the peritrochanteric structures and the pathologic processes most likely responsible for symptomatology and dysfunction, the physician will be better equipped to provide effective long-term solutions for this common problem. ${ }^{10}$

The reason for raising these issues is that primary care physicians often have limited training in use of intrabursal injections and few have access to ultrasound guidance. If they are going to perform such procedures, they should undergo intensive training and be required to have ultrasound facilities in the office so that the outcomes of treatment for bursitis in primary practice are the same as those achieved by subspecialists.

\section{Disclosure}

The authors report no conflicts of interest in this work.

\section{References}

1. Wittich CM, Ficalora RD, Mason TG, Beckman TJ. Musculoskeletal injection. Mayo Clin Proc. 2009;84:831-836.

2. Bhagra A, Syed H, Takahashi PY. Efficacy of musculoskeletal injections by primary care providers in the office: a retrospective cohort study. Int J Gen Med. 2013;6:237-243.

3. Hannonen P, Möttönen T, Oka M. Palindromic rheumatism. A clinical survey of sixty patients. Scand J Rheumatol. 1987;16:413-420.

4. Thomeé R, Augustsson J, Karlsson J. Patellofemoral pain syndrome: a review of current issues. Sports Med. 1999;28:245-262.

5. Brantingham JW, Cassa TK, Bonnefin D, et al. Manipulative therapy for shoulder pain and disorders: expansion of a systematic review. J Manipulative Physiol Ther. 2011;34:314-346.

6. Tortolani PJ, Carbone JJ, Quartararo LG. Greater trochanteric pain syndrome in patients referred to orthopedic spine specialists. Spine J. 2002;2:251-254. 
7. Jolly M, Curran JJ. Underuse of intra-articular and periarticular corticosteroid injections by primary care physicians: discomfort with the technique. J Clin Rheumatol. 2003;9:187-192.

8. De Carli A, Vadalà A, Perugia D, et al. Shoulder adhesive capsulitis: manipulation and arthroscopic arthrolysis or intra-articular steroid injections? Int Orthop. 2012;36:101-106.
9. Gasparre G, Fusaro I, Galletti S, Volini S, Benedetti MG. Effectiveness of ultrasound-guided injections combined with shoulder exercises in the treatment of subacromial adhesive bursitis. Musculoskelet Surg. 2012;96 Suppl 1:S57-S61.

10. Ho GW, Howard TM. Greater trochanteric pain syndrome: more than bursitis and iliotibial tract friction. Curr Sports Med Rep. 2012;11:232-238.

International Journal of General Medicine

\section{Publish your work in this journal}

The International Journal of General Medicine is an international, peer-reviewed open-access journal that focuses on general and internal medicine, pathogenesis, epidemiology, diagnosis, monitoring and treatment protocols. The journal is characterized by the rapid reporting of reviews, original research and clinical studies across all disease areas.

\section{Dovepress}

A key focus is the elucidation of disease processes and management protocols resulting in improved outcomes for the patient. The manuscript management system is completely online and includes a very quick and fair peer-review system. Visit http://www.dovepress.com/ testimonials.php to read real quotes from published authors.

Submit your manuscript here: http://www.dovepress.com/international-journal-of-general-medicine-journal 